\title{
CORRIGENDUM
}

\section{Low bone mass in premenopausal chronic dieting obese women}

\section{Bacon et al}

European Journal of Clinical Nutrition (2004) 58, 1326. doi:10.1038/sj.ejen.1602041

Correction to: European Journal of Clinical Nutrition (2004) 58, 966-971. doi:10.1038/sj.ejcn.1601922

Following the publication of the above paper, the authors have identified an error in the abstract and would like to apologize for any confusion this might have caused. A statement of the authors is reproduced below:

The abstract of the above article incorrectly stated that the data were gathered on women enrolled in a weight loss program. In actuality, the women had been recruited for a 'wellness program'. They were eventually randomized into two groups: one a weight loss intervention, and the other a 'health at every size' intervention intended to encourage healthy lifestyle behaviors without a focus on weight.

This mistake does not affect the data or outcomes presented in the paper, but does mischaracterise our future work. We apologize for the error. 